\title{
Cross-pollinating fields
}

\section{Enhanced crosstalk between plant and chemical biologists will lead to major advances in our molecular understanding of plant biology.}

P

lant biology has grown from its origins in botany to become an increasingly molecular science. Chemical biology, in turn, is evolving from its chemical roots to explore biological systems of greater complexity. Each discipline offers concepts and tools that will benefit the other, and we urge enhanced future collaboration between these two communities. To encourage the conversation, this issue contains a collection of articles that highlight emerging frontiers in plant chemical biology, with an emphasis on the networks of plant hormones that regulate plant growth and mediate plant interactions with their external environments.

Plant-derived small molecules are an important historical link between plant biology and chemical biology. Plants produce highly diverse collections of natural products, including alkaloids, polyketides and terpenoids, which have fascinated chemists for decades. Ongoing efforts to uncover new plant secondary metabolites have inspired new chemical methodologies and total synthesis approaches and have provided lead compounds for drug discovery efforts. Indeed, metabolic engineering for the large-scale production of natural plant metabolites such as alkaloids and non-natural analogs is an ongoing effort in chemical biology (p. 292).

Though these metabolites provide inspiration for chemists, plants make small molecules because they are an elegant solution to the problems imposed by their sessile lifestyle. Plants require effective mechanisms to transmit physiological signals between cells and throughout plant tissues. In addition, they must respond to changing environmental conditions, defend themselves against predation and infection, and communicate with beneficial members of their ecosystems. Small molecules are ideally suited for these tasks: stable metabolites available from biosynthetic pathways can bind to specific cellular targets and are easily mobilized for transport within the plant or for exchange with the environment.

Plant hormones are an important class of plant metabolites that feature prominently in this special issue. These hormones include a diverse collection of small molecules that are involved in all aspects of plant growth, development and defense, and research over the past decade has greatly expanded our understanding of these essential signaling molecules. Elucidation of plant hormone biosynthetic pathways and identification of their protein receptors and effectors have provided insights into how hormones elicit their biological functions (p. 301). In addition, the mechanisms used to transport plant hormones and other molecules, shown most clearly with the plant growth hormone auxin, are now coming to light ( $\mathrm{p}$. 325). Taken together, these studies reveal the emerging picture that plants use a network of plant hormone signaling and transport pathways to regulate growth and development within individual plants. Environmental stimuli then 'plug in' to this network (p. 308) and fine-tune physiological responses of plants to external factors such as pathogens (p. 308), herbivores (p. 317) and abiotic stressors (p. 283).

Understanding the molecular basis of this complex web of plant hormone pathways will require substantial experimental input from chemical biologists. First, elucidation of biosynthetic pathways leading to plant hormones plays to the strengths of many chemical biologists and is likely to reveal new enzymes and biochemical transformations (p. 268, p. 292). Second, tools from synthetic and analytical chemistry will provide increased chemical rigor in plant hormone signaling studies. For example, in this issue, Fonseca et al. (p. 273, p. 344) use a chemical approach to demonstrate that (+)-7-iso-jasmonyl-L-isoleucine is the primary bioactive jasmonate hormone and suggest a plausible epimerization mechanism for inactivation of this hormone in plants. Third, chemical biology efforts will likely uncover new regulatory small molecules, such as the recent discovery of strigolactone as a plant hormone involved in shoot branching (Nature 455, 189-194 and 195-200, 2008).

Chemical biology's arsenal of chemical tools will be essential in addressing other open questions in plant biology. Small molecules are routinely used by plant biologists to perturb plant metabolism, but there is a great need for more diverse classes of chemical probes that will target a broader cross section of plant pathways. Technologies for high-throughput screening of chemical libraries in plant systems need to be developed and used more widely in the plant biology community. The resulting probes offer a complementary approach to current genetic methods, particularly in model organisms such as Arabidopsis thaliana, where genomic information and collections of mutant strains are available (p. 268). Beyond small molecules, other chemical biology approaches that have demonstrated success in animal cells should be applied more broadly in plants. Chemical methods involving labeling or measurement of cellular components in vivo offer great potential for providing quantitative molecular data in plant systems. For example, new metal ion sensors could substantially advance our understanding of metal homeostasis and trafficking in plants (p. 333). Proteomic strategies, including chemical tools such as activity-based probes (Nat. Chem. Biol. $4,557-563,2008)$, have great potential to identify important molecular players in plant pathways. These molecular strategies, combined with genetics, cell biology and systems biology approaches, are likely to revolutionize the way we understand plant biology.

To develop these potential synergies, plant biologists and chemical biologists need to open the lines of communication and initiate collaborations on the many important scientific problems at the interface of these disciplines. Particularly in this climate of increased support for science in the United States, plant and chemical biologists should seek expanded resources and infrastructure to foster collaborations and large-scale community efforts at this interface (p. 268).

We hope that the articles in this issue will be both a resource for chemical biologists who seek to learn more about plant chemical biology and an inspiration for plant and chemical biologists to work more closely together to reveal what are sure to be fascinating molecular details underpinning plant biology. 